\title{
Tuberculosis y síndrome inflamatorio de reconstitución inmunológica. Caso pediátrico
}

Tuberculosis and immune reconstitution inflammatory syndrome. Pediatric case

\author{
Dra. Ana C. Bustos y Dra. Gabriela M. Záratea
}

\begin{abstract}
RESUMEN
La tuberculosis es una enfermedad muy frecuente en nuestro medio. A pesar de que la detección precoz y el tratamiento adecuado logran la curación en la mayoría de los pacientes, la dificultad en el diagnóstico, el abandono del tratamiento y la aparición de resistencia a los fármacos tradicionales generan que, en la actualidad, continúe siendo un importante problema de salud pública. En la Argentina, la tasa de morbilidad es de $25 / 100000$ habitantes, con un leve aumento en la mortalidad. Se presenta el caso de una paciente pediátrica con tuberculosis, que tuvo múltiples complicaciones asociadas a la enfermedad y a su tratamiento, entre las cuales se incluye el síndrome inflamatorio de reconstitución inmunológica, también conocido como reacción paradojal al tratamiento antituberculoso. Este representa una consecuencia clínica adversa al restablecimiento de la inmunidad en el paciente que padece una infección sistémica grave, como la tuberculosis miliar.

Palabras clave: meningitis tuberculosa, tuberculosis, síndrome inflamatorio de reconstitución inmune.
\end{abstract}

\begin{abstract}
Tuberculosis is a very frequent disease in our environment. Although early detection and adequate treatment achieve cure in most patients, the difficulty in diagnosis, the abandonment of treatment and the appearance of resistance to traditional drugs generate that at present it continues to represent an important public health problem. In Argentina, the morbidity rate is $25 / 100,000$ inhabitants, with a slight increase in mortality. We present the case of a pediatric patient with tuberculosis and multiple complications associated with the disease and its treatment. One of these complications was the immune reconstitution inflammatory syndrome or paradoxical reaction to antituberculosis treatment. It represents an adverse clinical consequence of the restoration of immunity in the patient suffering from a serious systemic infection such as miliary tuberculosis. Key words: tuberculous meningitis, tuberculosis, immune reconstitution inflammatory syndrome.
\end{abstract}

http: / / dx.doi.org/ 10.5546/ aap.2020.e39

a. Cuidados Intermedios y Moderados, Hospital Nacional de Pediatría "Prof. Dr. Juan P. Garrahan",

Ciudad Autónoma de Buenos Aires.

Correspondencia:

Dra. Ana C. Bustos: anaclarabustos@hotmail.com

Financiamiento: Ninguno.

Conflicto de intereses: Ninguno que declarar.

Recibido: 10-1-2019

Aceptado: 15-7-2019
Cómo citar: Bustos AC, Zárate GM. Tuberculosis y síndrome inflamatorio de reconstitución inmunológica. Caso pediátrico. Arch Argent Pediatr 2020;118(1):e39-e42.

\section{INTRODUCCIÓN}

La tuberculosis es una enfermedad infectocontagiosa crónica provocada por el bacilo Mycobacterium tuberculosis. La vía principal de contagio es la inhalación de microgotas expelidas al toser, hablar y respirar. Los síntomas en los niños son solapados. Los más frecuentes son tos, pérdida de peso, fiebre prolongada, hemoptisis, astenia, entre otros. El diagnóstico requiere una anamnesis detallada acerca de los posibles contactos, la baciloscopía directa y el cultivo de muestras, y la prueba tuberculínica (purified protein derivative; PPD, por sus siglas en inglés), que indica el contacto del paciente con el bacilo. El tratamiento tiene una duración de seis a doce meses de acuerdo con el compromiso inicial de la enfermedad.

La tuberculosis meníngea es una de las formas más graves de presentación de tuberculosis extrapulmonar con elevado índice de secuelas neurológicas en los sobrevivientes. La diseminación puede darse por vía hematógena o por contigüidad.

El síndrome inflamatorio de reconstitución inmunológica (SIRI) o reacción paradojal al tratamiento antituberculoso (RP) es una respuesta paradójica durante el tratamiento antituberculoso en la cual se evidencia un empeoramiento clínico y radiológico, en ocasiones, con la aparición de nuevas lesiones. Ha sido poco reconocido en niños inmunocompetentes, a pesar de presentar una incidencia considerable.

\section{CASO CLÍNICO}

Paciente de 6 años de edad, sin antecedentes de relevancia, que consultó a Urgencias por fiebre y vómitos de cinco días de evolución. Se encontraba en regular estado general, reactiva, con signos de hipoperfusión. Se auscultaban crepitantes en la base pulmonar derecha. Con el diagnóstico de sepsis de foco respiratorio, se 
realizó la expansión con cristaloides y se inició el tratamiento con ceftriaxona. La radiografía de tórax mostró un foco de consolidación en la base pulmonar derecha (Figura 1). En el análisis de laboratorio, se destacaba linfopenia, anemia e hiperplaquetosis. Los hemocultivos y la $P P D$ resultaron negativos.

En la anamnesis, la madre refería haber padecido tuberculosis durante el embarazo y haber recibido tratamiento por un año. Relataba condiciones habitacionales precarias y hacinamiento. Una vecina con quien compartían la habitación se encontraba en tratamiento contra la tuberculosis. A horas del ingreso, la paciente presentaba deterioro del sensorio y signos de irritación meníngea. Considerando los antecedentes y ante la imposibilidad de realizar la punción lumbar, se amplió el tratamiento empírico para meningoencefalitis con aciclovir, isoniacida, rifampicina, pirazinamida, etambutol y dexametasona.

Se realizó una resonancia magnética nuclear (RMN) de cerebro, que informó imágenes compatibles con leptomeningitis frontal con predominio derecho, edema y cerebritis del parénquima subyacente (Figura 2). Con sospecha de absceso cerebral, se amplió el esquema con meropenem. Se realizó una punción lumbar, que informó glucorraquia de $22 \mathrm{mg} \%$, proteinorraquia de $122 \mathrm{mg} / \mathrm{dl}$, 55 células $/ \mathrm{mm}^{3}$ con predominio neutrofílico. El cultivo del líquido cefalorraquídeo (LCR) fue negativo para gérmenes comunes.

FIGURA 1. Radiografía de tórax al ingresar con imagen de consolidación paracardíaca derecha con broncograma aéreo e infiltrado bilateral difuso

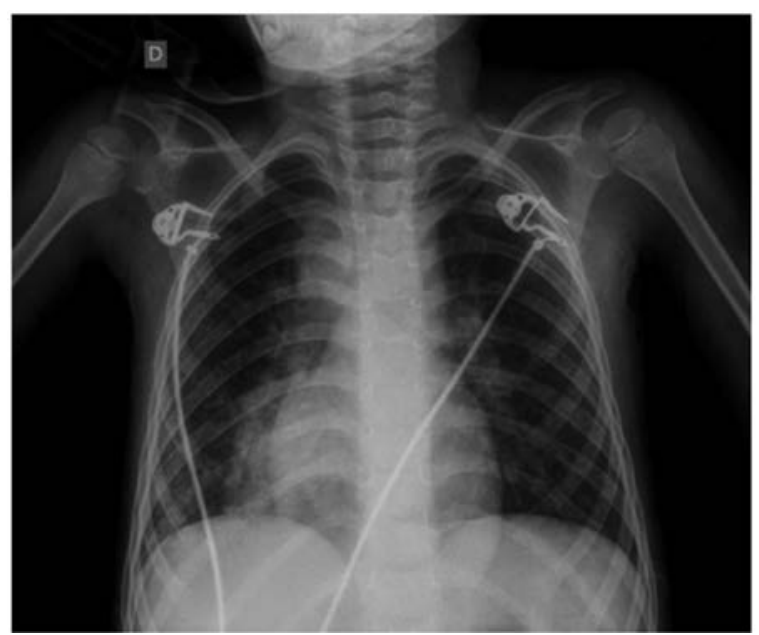

A las 3 semanas, con franca mejoría clínica, se suspendieron los antibióticos; se continuó con tuberculostáticos y se inició el descenso de corticoides. La niña evolucionó con síndrome febril, que se extendió por 4 semanas, sin cambios significativos al momento del examen físico. Se recibió el cultivo positivo para Mycobacterium tuberculosis en lavados gástricos, y se confirmó la sensibilidad del germen a las 4 drogas utilizadas.

La RMN de cerebro no evidenció cambios significativos respecto a las previas. En la tomografía de tórax y abdomen, se observaron nuevas imágenes caseificadas en el mediastino y los lóbulos pulmonares, con consolidación y atelectasia. En el hígado, imágenes hipodensas en segmento 4; en el bazo, múltiples imágenes de características similares a las hepáticas (Figura 3). En la RMN de columna, se observaron imágenes nodulares que se realzaban con el contraste compatibles con el impacto del proceso infeccioso a nivel espinal.

Ante el empeoramiento clínico, se corroboró la sensibilidad del bacilo, la correcta administración de los fármacos, se optimizó la

FIgURA 2. Resonancia magnética al ingresar

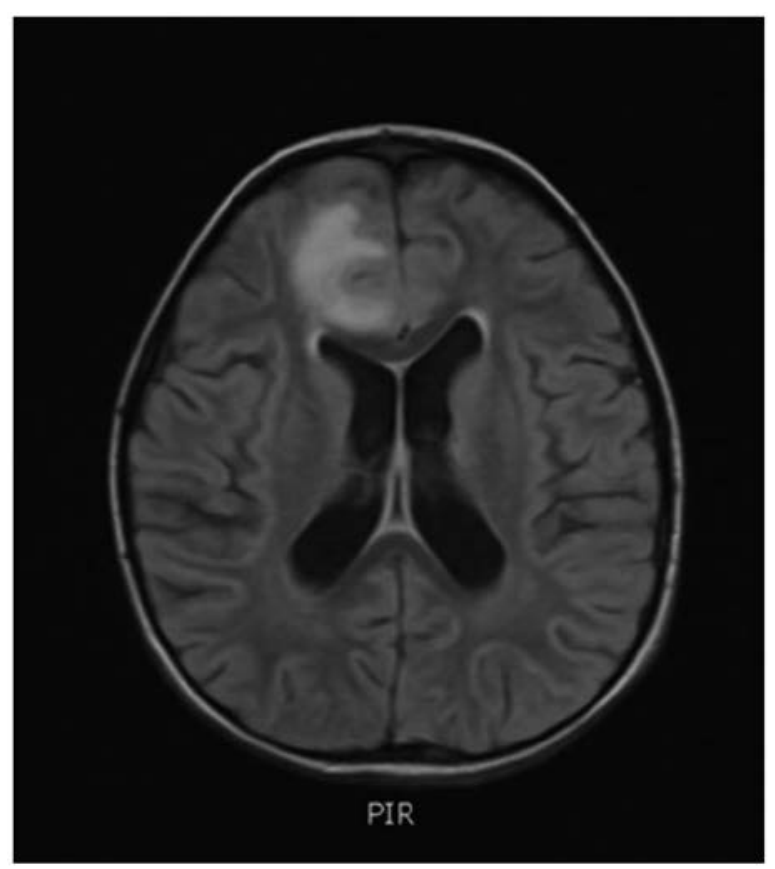

Resonancia magnética del sistema nervioso central al ingresar. T1. Marcado refuerzo leptomeníngeo con contraste en la región frontal medial con predominio derecho, asociado a señales corticosubcorticales hipointensas con leve efecto de masa, compatibles con leptomeningitis de probable etiología tuberculosa, asociada a edema y/o cerebritis del parénquima subyacente. Leve hidrocefalia comunicante. 
vía de administración y se descartaron infecciones agregadas. Interpretándose el cuadro como SIRI o RP en una paciente con tuberculosis miliar, se reinició el tratamiento con metilprednisona y evolucionó favorablemente. Se otorgó el egreso hospitalario para continuar el tratamiento prolongado con tuberculostáticos y corticoides.

Nuestra paciente presentó un cuadro clínico progresivo y grave. Dado el contacto fehaciente con tuberculosis, el tratamiento empírico se realizó tempranamente. Desarrolló SIRI como complicación asociada. Cumplió 2 meses de tratamiento con 4 drogas y 10 meses con isoniacida y rifampicina. Se realizó el descenso de corticoides sin reaparición de los síntomas y con mejoría de las lesiones en la RMN. Continuó el seguimiento ambulatorio sin presentar secuelas significativas.

\section{DISCUSIÓN}

Actualmente, la tuberculosis constituye la novena causa de muerte a nivel mundial y la primera por enfermedades infecciosas. En 2016,

FIgURA 3. Tomografía de abdomen

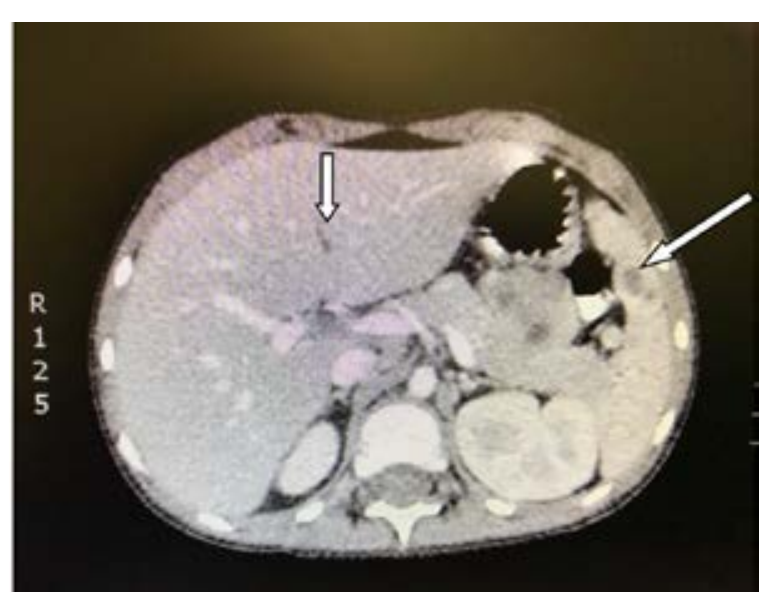

Hígado aumentado de tamaño, con dos imágenes hipodensas en el segmento IV. Bazo heterogéneo con múltiples imágenes redondeadas hipodensas. Adenopatías múltiples en el hilio hepático y esplénico. se notificaron 11560 nuevos casos en la Argentina, que correspondieron en un $17 \%$ a la población pediátrica. ${ }^{1}$

El compromiso pulmonar es la forma típica de presentación. Entre las formas extrapulmonares, la infección del sistema nervioso central representa uno de los focos con mayor morbimortalidad. En 2014, fueron notificados 32 casos de tuberculosis de sistema nervioso central en niños menores de 5 años en la Argentina. ${ }^{2}$ Suele presentarse como síndrome meníngeo de evolución subaguda y progresiva asociado a síntomas constitucionales. Otras formas de presentación incluyen el absceso cerebral, los tuberculomas, las trombosis vasculares e hidrocefalia. Alrededor del $50 \%$ de los niños con tuberculosis meníngea presentarán, al mismo tiempo, alteraciones en la radiografía de tórax. ${ }^{3}$

El LCR es la principal herramienta diagnóstica. Característicamente, presenta hipercelularidad $\left(50-500 / \mathrm{mm}^{3}\right)$, con predominio mononuclear, hiperproteinorraquia (200-5000 $\mathrm{mg} / \mathrm{dl})$ e hipoglucorraquia ( $<50 \%$ de la concentración sérica). Su normalización es esperable hacia los seis meses de tratamiento. El gold standard para la confirmación del diagnóstico es el cultivo del LCR. Los métodos de detección molecular por reacción en cadena de la polimerasa tienen una sensibilidad del $56 \%$ y una especificidad del $98 \%{ }^{4}$ Los estudios por imágenes de mayor utilidad son la tomografía computada y la RMN con contraste (esta última, de elección). ${ }^{5,6}$

El tratamiento de la meningitis tuberculosa se basa en un esquema inicial (fase intensiva) de cuatro fármacos (isoniacida, rifampicina, pirazinamida y etambutol) por 2 meses, seguidos de una fase de continuación de isoniacida y rifampicina por 7-10 meses. ${ }^{1} \mathrm{El}$ tratamiento con corticoides ha demostrado reducir la incidencia de muerte (riesgo relativo -RR-: 0,75, intervalo de confianza -IC- del $95 \%=0,65-0,87)$ y déficit neurológico residual en pacientes inmunocompetentes. Se recomienda dexametasona (0,3-0,4 mg/ kg/día) o prednisona (1-2 mg/kg/día). ${ }^{7}$

TABLA 1. Estadios clínicos de meningitis tuberculosa propuestos por el British Medical Research Council

\begin{tabular}{lll}
\hline Estadio I & \multicolumn{1}{c}{ Estadio II } & \multicolumn{1}{c}{ Estadio III } \\
\hline Síndrome infeccioso. Fiebre irregular. & Somnolencia alternada con irritabilidad. & Somnolencia, coma, \\
Vómitos 1-2 veces / día & Cefalea intensa, signos meníngeos. & signos meníngeos francos. \\
con 2-3 semanas de evolución. & Crisis convulsivas, & Hipertonía con opistótonos. \\
& afección de pares craneales. & Enclavamiento de amígdalas. \\
Mortalidad: el 1-3 $\%$. & Mortalidad: el 4,7-20,7 \%. & Mortalidad: el 21,9-48,9\%. \\
Secuelas neurológicas: el 26\% (8,7-58,5). & Secuelas: el 40,8 \% (27,8-57,3). & Secuelas: el 70,5\% (51,3-84,5). \\
\hline
\end{tabular}


El pronóstico está relacionado con el estadio de la enfermedad al momento del diagnóstico e inicio del tratamiento. La morbimortalidad aumenta notablemente cuando el niño presenta compromiso grave de la conciencia, mientras que, una vez iniciado el tratamiento, en las primeras fases, puede observarse una recuperación neurológica ad integrum (Tabla 1). Entre las secuelas se incluyen la alteración de pares craneales, convulsiones, trastornos psiquiátricos, ataxia, alteraciones cognitivas y retardo mental. ${ }^{8}$

El SIRI constituye un cuadro clínico de deterioro progresivo, coincidente con la restauración de la inmunidad en pacientes con afectación de esta como consecuencia de infecciones sistémicas graves. Es una respuesta inmune exagerada, paradojal al iniciar el tratamiento específico, con empeoramiento en los hallazgos clínicos e imagenológicos. Se ha descrito ampliamente en pacientes con tuberculosis e infección por el virus de la inmunodeficiencia humana al iniciar ambos tratamientos, con incidencias entre el $16 \%$ y el $31 \% .{ }^{9}$ Sin embargo, también puede presentarse en pacientes inmunocompetentes secundario a la infección por citomegalovirus, Pneumocystis carinii y herpes.

El diagnóstico de SIRI se establece por exclusión. Ante el empeoramiento clínicoradiológico en un paciente en tratamiento contra tuberculosis, se debe descartar su incumplimiento, el desarrollo de resistencia de la cepa, errores en la dosificación y en la forma de administración, y la aparición de nuevos procesos infecciosos o inmunológicos que pudieran justificar los síntomas. No han sido establecidos criterios diagnósticos para pacientes inmunocompetentes hasta la actualidad. ${ }^{9}$

La incidencia de SIRI fue reportada en 3 estudios retrospectivos entre el 9,8\% y el $15 \%{ }^{11-13}$ en niños con diagnóstico de tuberculosis. El estado nutricional deficiente, la menor edad, el compromiso grave inicial, el bajo recuento linfocitario y la PPD negativa han sido asociados como factores de riesgo para desarrollar SIRI. ${ }^{11-13}$

El tiempo descrito entre la aparición de los síntomas de SIRI y el inicio del tratamiento antituberculoso se estimó en 42 días (23-53 días), con una expectativa de resolución clínica de 10 días (3-15 días) y radiológica de 45 días (26-105 días). ${ }^{10-13}$ Esta resolución suele ser espontánea, aunque, en algunos casos, ante el compromiso grave, se recomiendan ciclos de corticoides para suprimir las reacciones inflamatorias exageradas (metilprednisona en dosis de $1 \mathrm{mg} / \mathrm{kg} /$ día) ${ }^{10}$ por 4-6 semanas.
La tuberculosis es una enfermedad prevenible, con una alta prevalencia en nuestro país que obliga a tenerla siempre presente como diagnóstico diferencial ante diversas patologías. Es fundamental la vacunación, el diagnóstico y el tratamiento precoz, y el estudio de contactos para detectar el caso índice y cortar la cadena de contagio. El diagnóstico de SIRI requiere alta sospecha y se basa en parámetros clínicos que reflejan un empeoramiento de la infección o una inflamación sistémica importante secundaria al inicio del tratamiento.

\section{REFERENCIAS}

1. World Health Organization. Global tuberculosis report. Geneva: WHO; 2016. [Acceso: 16 de julio de 2019]. Disponible en: https: / / apps.who.int/iris/bitstream/han dle / 10665/250441/9789241565394-eng.pdf;jsessionid=59 AC374E63C2865223B87415ECE6CF7E?sequence=1.

2. Tuberculosis. Guía para el equipo de salud. Nro. 3. 2. da ed. Buenos Aires: Ministerio de Salud de la Nación; 2014. [Acceso: 16 de julio de 2019]. Disponible en: http: / / www. msal.gob.ar/images/stories/bes/graficos/0000000049cntguia_de_diagnostico_tratamiento_y_prevencion_de_la_ tuberculosis_2015.pdf.

3. Solomons RS, Goussard P, Visser DH, Marais BJ, et al. Chest radiograph findings in children with tuberculous meningitis. Int J Tuberc Lung Dis. 2015; 19(2):200-4.

4. Pai M, Flores LL, Pai N, Hubbard A, et al. Diagnostic accuracy of nucleic acid amplification tests for tuberculous meningitis: a systematic review and meta-analysis. Lancet Infect Dis. 2003; 3(10):633-43.

5. Gupta RK, Kumar S. Central nervous system tuberculosis. Neuroimaging Clin N Am. 2011; 21(4):795-814.

6. Helmy A, Antoun N, Hutchinson P. Cerebral tuberculoma and magnetic resonance imaging. J $R$ Soc Med. 2011; 104(7):299-301.

7. Prasad K, Singh MB, Ryan H. Corticosteroids for managing tuberculous meningitis. Cochrane Database Syst Rev. 2016; 4:CD002244.

8. Chiang SS, Khan FA, Milstein MB, Tolman AW, et al. Treatment outcomes of childhood tuberculous meningitis: A systematic review and meta-analysis. Lancet Infect Dis. 2014; 14(10):947-57.

9. Meintjes G,LawnSD,ScanoF,Maartens G, etal. Tuberculosisassociated immune reconstitution inflammatory syndrome: case definitions for use in resource-limited settings. Lancet Infect Dis. 2008; 8(8):516-23.

10. Murthy AR, Marulappa R, Hegde U, Kappadi D, et al. Treatment guidelines and prognosis of immune reconstitution inflammatory syndrome patients: a review. J Int Oral Health. 2015; 7(4):92-5.

11. Thampi N, Stephens D, Rea E, Kitai I. Unexplained deterioration during antituberculous therapy in children and adolescents: clinical presentation and risk factors. Pediatr Infect Dis J. 2012; 31(2):129-33.

12. Olive C, Mouchet F, Toppet V, Haelterman E, et al. Paradoxical reaction during tuberculosis treatment in immunocompetent children: clinical spectrum and risk factors. Pediatr Infect Dis J. 2013; 32(5):446-9.

13. Carazo Gallego B, Moreno-Pérez D, Nuñez Cuadros E, Mesa Fernández A, et al. Paradoxical reaction in immunocompetent children with tuberculosis. Int J Infect Dis. 2016 ; $51: 15-8$. 